\title{
Erratum zu: Allergien auf Schaben, Zecken, Vorratsmilben und andere Gliederfüßer: Wie weit ist die molekulare Allergiediagnostik? Teil 15 der Serie Molekulare Allergologie
}

Englische Fassung http://link.springer. com/journal/40629
Erratum zu: Hilger C, Kuehn A, Raulf M, Jakob T. Cockroach, tick, storage mite and other arthropod allergies: Where do we stand with molecular allergy diagnostics? Part 15 of the Series Molecular Allergology. Allergo J Int 2014;23:172-8

\section{DOI: 10.1007/s40629-014-0024-2}

In Tabelle 3 „Identifizierte Einzelallergene anderer Arthropoden gemäß IUIS Allergen Nomenclature Sub-Committee“ auf S. 176 hätte im Abschnitt „Chironomus thummi thummi (Rote Mückenlarve)" der Hämoglobinkomponente I/IA das Allergen Chi t 2, nicht Chi t 1 zugeordnet werden müssen. Nachfolgend ist der Abschnitt aus Tabelle 3 nochmals komplett in korrigierter Form wiedergegeben.

\begin{tabular}{|c|c|c|}
\hline \multicolumn{3}{|c|}{$\begin{array}{l}\text { Identifizierte Einzelallergene anderer Arthropoden } \\
\text { gemäß IUIS Allergen Nomenclature Sub-Committee }\end{array}$} \\
\hline Allergen & Name & Molekulargewicht (kD) \\
\hline \multicolumn{3}{|l|}{$\begin{array}{l}\text { Chironomus thummi } \\
\text { thummia, }, \mathrm{d}, \mathrm{e}, \mathrm{f} \\
\text { (Rote Mückenlarve) }\end{array}$} \\
\hline Chit 1 & Hämoglobinkomponente III/IV & 16 \\
\hline Chit 2 & Hämoglobinkomponente I/IA & 16 \\
\hline Chit 3 & $\begin{array}{l}\text { Hämoglobinkomponenten II- } \beta, \mathrm{VI} \text {, } \\
\text { VIII, IX }\end{array}$ & 16 \\
\hline Chit 4 & Hämoglobinkomponente IIIA & 16 \\
\hline Chit 9 & Hämoglobinkomponente $\mathrm{X}$ & 16 \\
\hline
\end{tabular}

\section{Christiane Hilger, PhD}

Annette Kuehn

Laboratoire d'Immunogénétique et d'Allergologie

Centre de Recherche Public de la Santé, Luxembourg, Luxembourg

E-Mail: christiane.hilger@crp-sante.lu

\section{Monika Raulf}

Institut für Prävention und Arbeitsmedizin der Deutschen Gesetzlichen Unfallversicherung, Institut der Ruhr-Universität Bochum (IPA), Deutschland

\section{Thilo Jakob}

Forschergruppe Allergologie, Klinik für Dermatologie und Venerologie, Universitätsklinikum Freiburg, Deutschland

\section{Zitierweise}

Hilger C, Kuehn A, Raulf M, Jakob T. Erratum to: Cockroach, tick, storage mite and other arthropod allergies: Where do we stand with molecular allergy diagnostics? Part 15 of the Series Molecular Allergology. Allergo J Int 2014:23:281

DOI: $10.1007 / \mathrm{s} 40629-014-0035-z$ 